Ger J Exerc Sport Res 2022 · 52:173-178 https://doi.org/10.1007/s12662-021-00757-z Eingegangen: 30. März 2021

Angenommen: 4. September 2021

Online publiziert: 4. Oktober 2021

(c) Der/die Autor(en) 2021

\author{
Dirk Büsch ${ }^{1}$ (D) - Till Utesch ${ }^{2}$. Franz Marschall ${ }^{3}$ \\ ${ }^{1}$ Carl von Ossietzky Universität, Oldenburg, Deutschland \\ ${ }^{2}$ Westfälische Wilhelms-Universität, Münster, Deutschland \\ ${ }^{3}$ Universität des Saarlandes, Saarbrücken, Deutschland
}

\title{
Entwicklung und Evaluation der Anstrengungsskala Sport
}

Unter dem Begriff ,internal load“ werden im Rahmen eines Monitorings der Beanspruchung in Training und Wettkampf sowohl objektive Parameter, z.B. die Herzfrequenz, als auch subjektive Parameter, z.B. die Selbsteinschätzung der wahrgenommenen Anstrengung verstanden. Bei der Messung dieser Selbsteinschätzung werden psychische und physische Beanspruchungen in einer subjektiven Bewertung zusammengefasst (Borg, 1982; Chen, Fan, \& Moe, 2002; Enoka \& Duchateau, 2016; Impellizzeri, Marcora, \& Coutts, 2019; Morgan, 1994). Die Selbsteinschätzung kann auf dieser theoretischen Grundlage altersund geschlechtsunabhängig, zuverlässig und valide bestimmt werden (Garcin, Fleury, Mille-Hamard, \& Billat, 2005; Lamb, Parfitt, \& Eston, 2017; Robertson et al., 2000). Als Erhebungsinstrumente existieren einerseits differenzierte Skalen mit mehreren Items (Hitzschke et al., 2016; Hitzschke et al., 2015; Kellmann, Kölling, \& Hitzschke, 2016; Kölling et al., 2015; Kölling \& Kellmann, 2020) und andererseits Ein-Item-Skalen, z.B. die sogenannte Borg- oder RPE-Skala (Rating of Perceived Exertion Scale, Borg, 1998). Aufgrund ihrer Einfachheit und Nutzerfreundlichkeit wird für die Erfassung der subjektiven Beanspruchungswahrnehmung in Training und Wettkampf bevorzugt die Category Ratio Scale (CR10-Skala) oder eine daran angelehnte Single-Item-Skala eingesetzt (Borg, 1998; Foster et al., 2021; Gaudino et al., 2015; Haddad, Stylianides, Djaoui, Dellal, \& Chamari, 2017), die ursprünglich aus der an der Herzfrequenz orientierten RPE-Skala abgeleitet und validiert wurde (๑Tab. 1, Borg,
1998; Löllgen, 2004). Die CR10-Skala stellt eine nicht lineare, leicht positiv beschleunigte Funktion bezogen auf die Beanspruchung dar (Eston \& Parfitt, 2006; Faulkner \& Eston, 2008), die in der von Borg autorisierten deutschen Fassung mit "Anstrengungsempfinden“ übersetzt wird (Borg, 2004; Löllgen, 2004). Pageaux (2016) und Fisher und Smith (2012) sowie Fisher, Steele, BruceLow und Smith (2011) haben aus unterschiedlichen Perspektiven darauf hingewiesen, dass die Begriffe "exertion“ und „intensity“ synonym zu "effort“ (Anstrengung) zu verstehen sind. Die Beanspruchungsintensität kann daher über den Anstrengungsgrad erfasst werden (Carton \& Rhodes, 1985; Lamb et al., 2008/2013; Noble \& Robertson, 1996). Darüber hinaus kann der effektive Workload, d.h. das Produkt aus Anstrengungsgrad und Dauer einer Trainingseinheit als Session-RPE für ein systematisches Monitoring eingesetzt werden (Foster et al., 2021; Foster et al., 2001; Foster, Rodriguez-Marroyo, \& de Koning, 2017; Gabbett, 2020; Gomes et al., 2020; Haddad, Padulo, \& Chamari, 2014; Haddad et al., 2017; Robertson et al., 2000; Scott, Duthie, Thornton, \& Dascombe, 2016).

Die CR10-Skala von Borg (1982), die im Original keine zehn-, sondern eine dreizehnstufige Skala mit Zwischenschritten darstellt, wurde mit der Intention entwickelt, dass „....numbers should be anchored by verbal expressions that are simple and understandable by most people. The expressions should in turn be placed in the correct position on a ratio scale, where the expressions belong according to their quantitative meaning" (S. 380). Des Weiteren wies Borg daraufhin, dass unabhängig von der Belastungsart die anstrengendste Übung mit „very, very strong/heavy" und das Gegenteil mit „very, very weak/light“ bezeichnet werden könnte. Es erscheint daher entgegen der ursprünglichen Zielstellung nicht verwunderlich, dass zahlreiche Versionen der CR10-Skala mit unterschiedlichen und überwiegend unvollständigen Stufenbezeichnungen existieren (Halperin \& Emanuel, 2020). Auch wenn semantische Unterschiede bei den verwendeten unterschiedlichen Stufenbezeichnungen, z. B. "hard, heavy, strong" anzunehmen sind, erscheint eine exakte Übersetzung und Diskussion der Stufenbezeichnungen nicht zielführend, da die Einschätzung der Anstrengung maßgeblich über den Kontext, d.h. über die konkrete Fragestellung und damit den situativen Bedeutungsgehalt bestimmt wird (Äquivalenzkriterium, Behr, Braun, \& Dorer, 2015). Dennoch ist sprachliche bzw. begriffliche Klarheit notwendig, um Überschneidungen mit

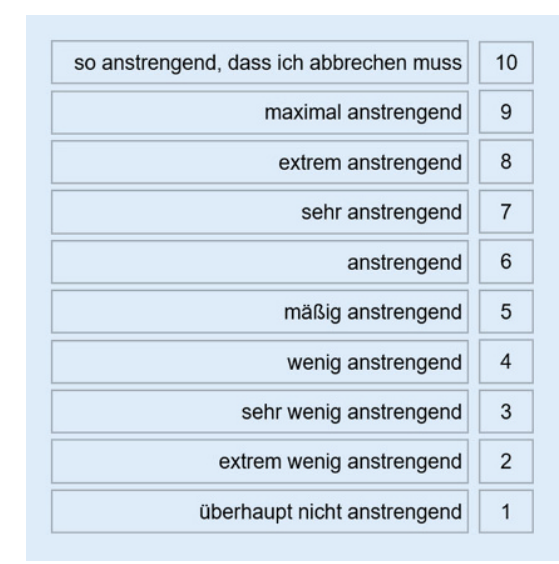

Abb. $1 \Delta$ Anstrengungsskala Sport (ASS) 


\section{Kurzbeitrag}

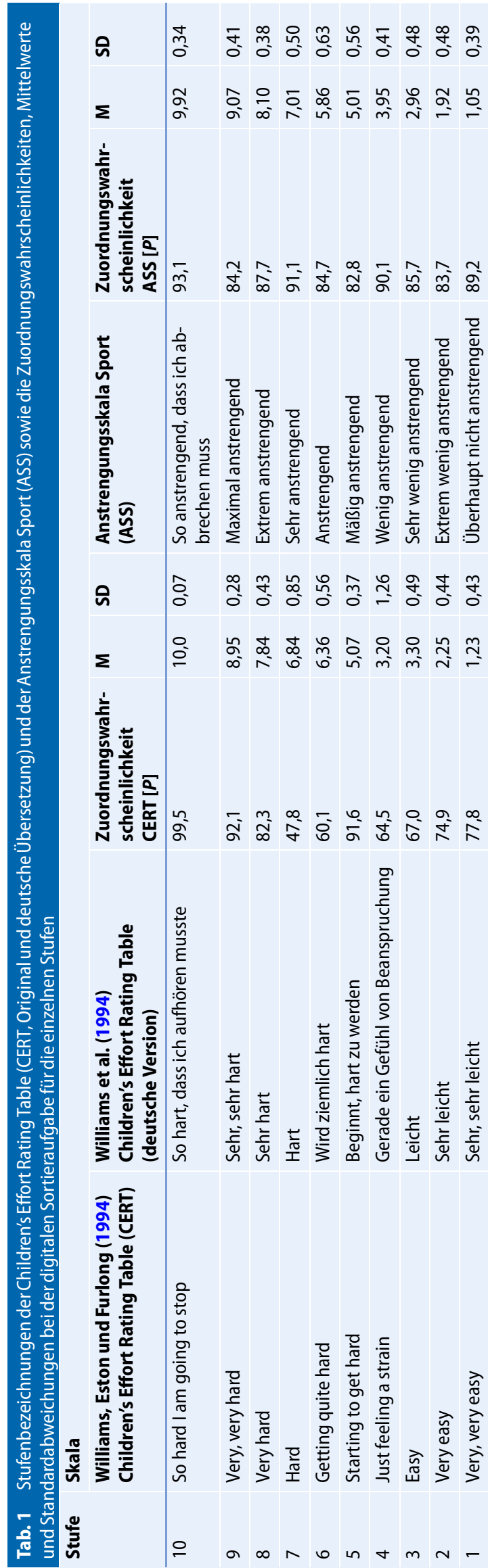

verwandten Konstrukten, z. B. Schmerz („pain“), Unbehagen („discomfort“), Kraft („force“) oder Ermüdung („fatigue") zu vermeiden (Abbiss, Peiffer, Meeusen, \& Skorski, 2015; Pageaux, 2016). Auch wenn der Bedeutungsgehalt maßgeblich über den Kontext bestimmt wird und die unterschiedlichen CR10Skalen im Monitoring bereits eingesetzt werden (können), erscheint die Entwicklung einer deutschsprachigen Skala mit vollständigen Stufenbezeichnungen sowie semantischer Klarheit unter Berücksichtigung der Zielsetzung von Borg (1982) dringend geboten, um Anstrengung zuverlässig und valide messen zu können.

\section{Entwicklung einer Anstrengungsskala}

In Anlehnung an die inhaltlichen Überlegungen von Borg (1982) sowie das paradigmatische, methodologische Vorgehen von Jonkisz, Moosbrugger, und Brandt (2012) soll das Konstrukt Anstrengung, d.h. die individuell wahrgenommene Beanspruchung bei einer sportlichen Belastung erhoben bzw. gemessen werden. Dafür wurde eine unipolare diskret gestufte Ein-Item-Ratingskala in Anlehnung an die CR10Skala für Sportlerinnen und Sportler entwickelt. Im ersten Schritt wurde von den Autoren eine rationale Konstruktionsstrategie in Anlehnung an Borg (1982) und Williams et al. (1994) gewählt. Der Aufgabenstamm lautet: „Wie anstrengend ist die Aufgabe (resp. Trainingseinheit) gewesen?“. Das Adjektiv anstrengend wird komparativ von überhaupt nicht bis so anstrengend, dass ich abbrechen muss durchdekliniert (Borg, 2004). Für die vorläufige verbale Ratingskala sowie die numerische Ratingskala von eins bis zehn wurde eine exhaustive Zuordnungsaufgabe erstellt, um die Eindeutigkeit der Item-Inhalte bzw. Bezeichnungen der Skalenstufen empirisch prüfen zu können. Im Rahmen einer Pilotstudie mit fünf Sportwissenschaftlerinnen und 15 Sportwissenschaftlern (Alter: 25-55 Jahre) sowie zehn jugendlichen Kaderathletinnen und 15 Kaderathleten (Alter: 16-18 Jahre) wurde die Zuordnungsaufgabe mit einer analogen 
Sortieraufgabe in einem individuellen Setting geprüft (Flick, 2002). Die Aufgabe bestand darin, zehn ungeordnet und gleichzeitig präsentierte Karteikarten (DIN A7, weiße Karten mit schwarzer Schrift) mit den Abstufungsbezeichnungen von überhaupt nicht anstrengend bis so anstrengend, dass ich abbrechen muss in Relation zu einer ansteigenden Beanspruchung anzuordnen. Zur Orientierung für eine ansteigende $\mathrm{Be}$ anspruchung bzw. aufsteigende Skala wurde die Stufenbezeichnung Ruhe als Ausgangsreferenz vorgegeben (Comyns \& Flanagan, 2013). Die Ergebnisse verdeutlichen, dass sowohl die Sportwissenschaftlerinnen und -wissenschaftler als auch die jugendlichen Kaderathletinnen und -athleten bei der Sortieraufgabe eine fast perfekt übereinstimmende Reihenfolge der verbalen und numerischen Ratingskala erreichten (Kaderathlet*innen $\operatorname{ICC}[2,1]=0,99 ; 95 \%$ KI 0,97-0,99; Sportwissenschaftler ${ }^{*}$ innen $\operatorname{ICC}[2,1]=99$, $95 \%$ KI 0,98-0,99), was zunächst als systematisch differenzierende, altersunabhängige und interindividuell vergleichbare Anstrengungseinschätzung interpretiert werden kann.

\section{Evaluation}

Für die Evaluation bzw. Item-Analyse wurde die vorläufige Testversion der Anstrengungsskala (ASS, - Abb. 1) sowie die Children's Effort Rating Table ${ }^{1}$ (CERT, Williams et al., 1994), die sich als eine der wenigen kontextbezogenen Skalen ebenfalls durch vollständige Stufenbezeichnungen auszeichnet, in einer anonymen Online-Umfrage mit sporttreibenden Erwachsenen im Alter von 19-40 Jahren $(M=24,2, S D=4,1)$ geprüft. An der Studie nahmen $N=244$ Personen teil, von denen $n=203(w=109, m=94)$ vollständige Datensätze berücksichtigt werden konnten. Die digitale Sortieraufgabe bestand darin, die gleichzeitig, aber ungeordnet und randomisiert präsentierten verbalen Stufenbezeichnungen einer auf-

\footnotetext{
1 Auch wenn die Skala ursprünglich für Kinder entwickelt wurde, wird sie aufgrund ihrer vollständigen Stufenbezeichnungen umfassend auch im Bereich der Anstrengungsdiagnostik bei Erwachsenen eingesetzt.
}

Ger J Exerc Sport Res 2022 ·52:173-178 https://doi.org/10.1007/s12662-021-00757-z (c) Der/die Autor(en) 2021

\section{Büsch · T. Utesch · F. Marschall}

\section{Entwicklung und Evaluation der Anstrengungsskala Sport}

\section{Zusammenfassung}

Aufgrund ihrer Einfachheit und Nutzerfreundlichkeit wird für die Erfassung der subjektiven Beanspruchungswahrnehmung in Training und Wettkampf bevorzugt die Category Ratio Scale (CR10-Skala) oder eine daran angelehnte Ein-Item-Skala eingesetzt. Die CR10-Skala stellt eine nichtlineare, leicht positiv beschleunigte Funktion bezogen auf die Beanspruchung dar, die in der autorisierten deutschen Fassung mit „Anstrengungsempfinden“ übersetzt wird. Allerdings ist festzuhalten, dass die existierenden Skalen keine vollständigen Stufenbezeichnungen beinhalten und/oder die Stufenbezeichnungen Überschneidungen mit verwandten Konstrukten, z. B. Ermüdung zulassen. Die Anstrengungsskala Sport (ASS) zeichnet sich demgegenüber durch vollständige Stufenbezeichnungen und begriffliche Klarheit mit der komparativen Deklination des Adjektivs anstrengend aus. Auf der Grundlage einer rationalen Konstruktionsstrategie sowie einer systematischen Item-Analyse kann gezeigt werden, dass die Voraussetzungen für die Verwendung der ASS als Verhältnisskala gegeben sind, die eine zuverlässige und inhaltlich eindeutige Messung der Anstrengung gestattet.

Schlüsselwörter

Beanspruchung · Skalenentwicklung .

Skalenevaluation $\cdot$ Monitoring

\section{Development and evaluation of the "Anstrengungsskala Sport" (Effort Scale Sport)}

\begin{abstract}
Due to its simplicity and user-friendliness, the Category Ratio Scale (CR10 scale) or a singleitem scale is preferred for recording subjective perception of internal load in training and competition. The CR10 scale represents a nonlinear, slightly positively accelerated function related to internal load, which is translated as "Anstrengungsempfinden" (effort) in the authorized German version. However, it should be noted that the existing scales do not include complete level descriptions and/or the level description allow overlap with related constructs, e.g., fatigue. In contrast, the "Anstrengungsskala Sport"
\end{abstract}

(Effort Scale Sport) is characterized by complete level descriptions and conceptual clarity with the comparative declension of the adjective strenuous. Based on a rational construction strategy as well as a systematic item analysis, it can be shown that the prerequisites for the use of the ASS as a ratio scale are given, which allows reliable and conceptually unambiguous measurement of effort.

\section{Keywords}

Internal load · Scale development · Scale evaluation - Monitoring steigenden Skala von 1-10 mit der Funktion Drag-and-Drop einzeln zu zuordnen. Beide Skalen wurden in randomisierter und ausbalancierter Reihenfolge präsentiert und von den Personen nacheinander bearbeitet.

Für die Bestimmung des Schwierigkeitsindex ergab eine korrekte Zuordnung den Wert 0 und eine fehlerhafte Zuordnung den Wert 1. Die Zuordnungswahrscheinlichkeit bzw. Item-Schwierigkeit für die einzelnen Stufen liegt für die ASS im Wertebereich zwischen $82,8 \leq P \leq 93,1$ und für die CERT im Wertebereich zwischen $47,8 \leq P \leq 99,5$ (- Tab. 1). Während bei der ASS die höchste Zuordnungswahrscheinlichkeit bei der Stufenbezeichnung „so anstrengend, dass ich abbrechen musste“ $(P=93,1)$ und die geringste bei der Stufe „mäßig anstrengend“ $(P=82,8) \mathrm{zu}$ konstatieren ist, erweist sich bei der CERT die höchste Zuordnungswahrscheinlichkeit bei der Stufe „so hart, dass ich aufhören musste" $(P=99,5)$ und die geringste bei der Stufe "hart“ $(P=47,8)$. Zusammenfassend erweisen sich die Zuordnungswahrscheinlichkeiten bzw. Item-Schwierigkeiten für die ASS konsistenter bzw. einfacher als bei der CERT. 

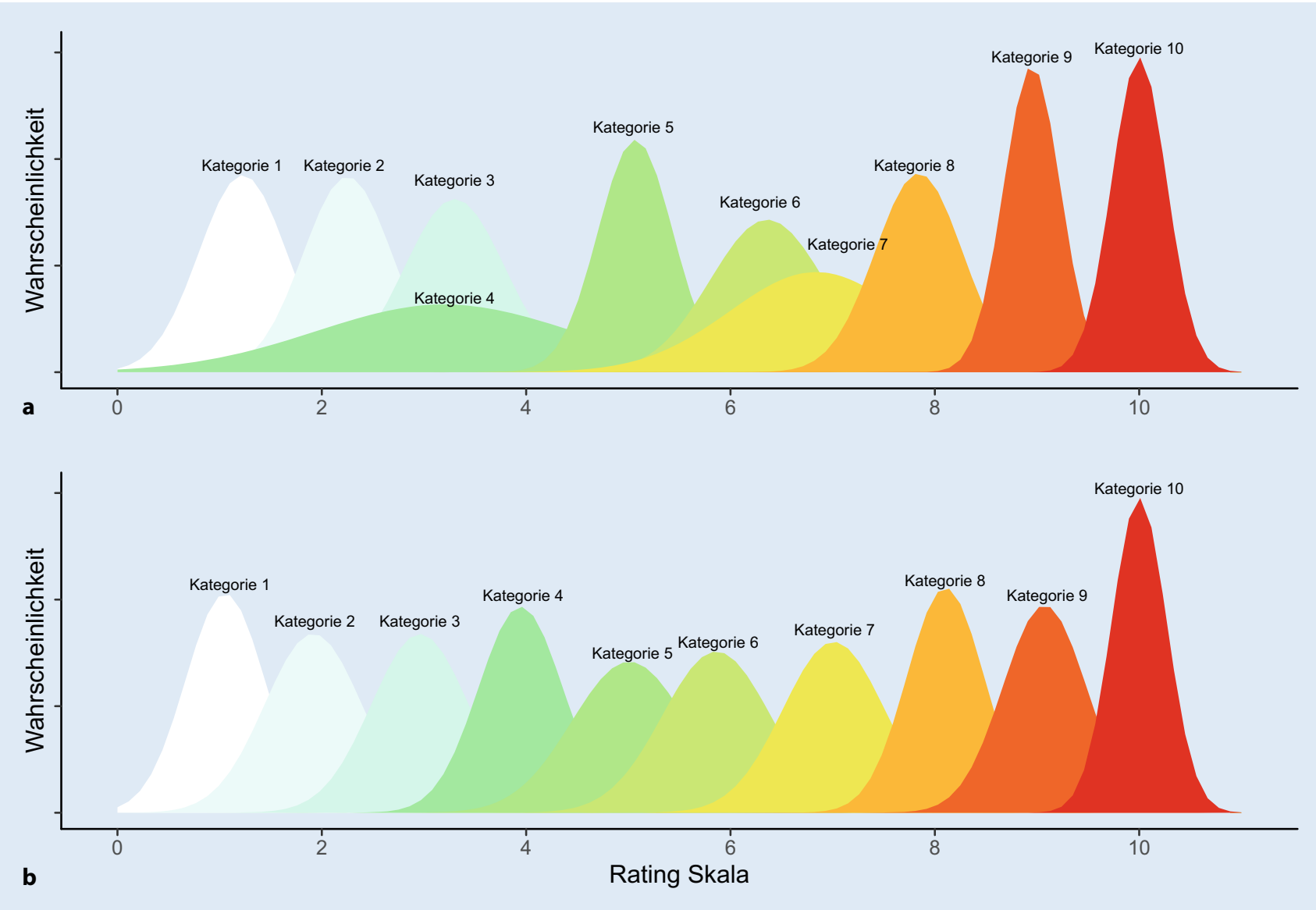

Abb. 2 \ Wahrscheinlichkeitsfunktionen für die einzelnen Stufen der Children's Effort Rating Table (CERT, a) sowie der Anstrengungsskala Sport (ASS, b)

CR10-Skalen sind jedoch als Verhältnisskalen konzipiert, sodass neben einem definierten Nullpunkt Äquidistanz zwischen den Skalenpunkten bzw. Stufenbezeichnungen gefordert sind. Die Forderung der Äquidistanzannahme wurde in Anlehnung an die Modelle der Item-Response-Theorie (IRT) geprüft. Hierfür wurden die Zuordnungen angelehnt an „item characteristic curves“ zum einen in Wahrscheinlichkeitsfunktionen für jede Stufe überführt und zum anderen die Schwellenabstände über die Mittelwertsdifferenzen zwischen den einzelnen Stufen berechnet. Zusammenfassend kann die Äquidistanz für die ASS aufgrund der gleichmäßigen und geordneten Wahrscheinlichkeitsverteilungen (• Abb. 2) der regelmäßigen Schwellenabstände $(0,85-1,15$; • Tab. 1) angenommen werden, wohingegen eine Äquidistanz für die CERT aufgrund der nicht durchgehend geordneten und ungleichmäßigen Wahrscheinlichkeits- verteilungen sowie der deutlich divergierenden Schwellenabstände $(-0,10-1,87)$ nicht gegeben scheint.

\section{Forschungsstand und weiterführende Überlegungen}

Single-Item-Skalen erlauben eine ökonomische und effiziente Messung von Merkmalen, bei denen Item-Rohwerte mit den Skalenwerten identisch sind, sodass die Objektivität als gegeben angesehen werden kann (Lienert \& Raatz, 1994). Die Anstrengungsskala Sport (ASS) stellt zum jetzigen Zeitpunkt lege artis eine Single-Item-Skala zur subjektiven Anstrengungseinschätzung dar, die durch die systematische Verwendung des Adjektivs „anstrengend“ auf jeder Stufe zum einen Überschneidungen mit ähnlichen Skalen, z. B. Ermüdung, Unwohlsein, Schmerz usw. (Halperin \& Emanuel, 2020; Micklewright, St Clair Gibson, Gladwell, \& Al Salman, 2017; Pa- geaux, 2016) vermeidet und zum anderen neben interindividuellen Unterschieden auch intraindividuelle Veränderungen und Effekte zuverlässig abbilden kann (Pageaux, 2016).

Der Einsatz der ASS erscheint bei sporttreibenden Erwachsenen, aber auch bei Sportanfängerinnen und -anfängern aufgrund der bisherigen $\mathrm{Da}$ tenlage gerechtfertigt, da wesentliche Erwartungen an empirisch geprüfte, qualitativ hochwertige, standardisierte Kurzskalen erfüllt sind (https://www. gesis.org/kurzskalen-psychologischermerkmale). Bisher nicht geprüfte Aspekte, z.B. zur divergenten Validität, sowie die Relevanz der ASS im Sinne eines Monitorings für das Erkennen von Übertrainingsmerkmalen und Verletzungsrisiken sind in weiteren Studien zu prüfen (Foster et al., 2021; Gabbett, 2016). Ebenso erscheinen Studien angezeigt, in denen bspw. der Einsatz bei Kindern und Jugendlichen (Eston, 
Lamb, Bain, Williams, \& Williams, 1994; Eston \& Parfitt, 2006; Kasai, Parfitt, Tarca, Eston, \& Tsiros, 2021; Lamb et al., 2017) geprüft und objektive Beanspruchungsgrößen für weitergehende Vergleiche herangezogen werden können (Borg, 2004; Egan, Winchester, Foster, \& McGuigan, 2006; Gomes et al., 2020; Hiscock, Dawson, Donnelly, \& Peeling, 2016; Hiscock, Dawson, \& Peeling, 2015; Mayo, Iglesias-Soler, \& Fernández-DelOlmo, 2014; Robertson et al., 2003). Des Weiteren wäre zu prüfen, inwieweit eine Farbzuordnung über gleichmäßige Abstufungen der Spektralfarben und der Farbintensität von Weiß (HSL: Farbton: 0, Sättigung: 255, Intensität: 128) über schwach hellblau, moderat hellgrün $\mathrm{zu}$ intensiv Rot (HSL: Farbton: 170, Sättigung: 0, Intensität: 255) als kongruent und unterstützend wahrgenommen wird („mode congruence“, Buckley \& Eston, 2000; Serafim et al., 2014).

Unter der Voraussetzung, dass (1) eine eindeutige Instruktion - z.B. „Wie anstrengend ist die Aufgabe (resp. Trainingseinheit) gewesen?" - verwendet wird, dass (2) eine Abstimmungs- bzw. Übungsphase für die Verwendung der Skala berücksichtigt wird und dass (3) unmittelbar nach einer Belastung, d. h. innerhalb von maximal $30 \mathrm{~min}$ nach dem Training oder des Wettkampfs, die Beanspruchung bspw. über eine App erfasst werden kann (Foster et al., 2021), stellt die Anstrengungsskala Sport (ASS) eine zuverlässige und sprachlich eindeutige Single-Item-Skala dar, die mehr Systematik in diesen wichtigen Bereich der Leistungssteuerung und des Monitorings bringen kann. Die Entwicklung aufgaben- bzw. kontextspezifischer Ankerbeispiele, insbesondere für den mittleren Anstrengungsbereich, sollte perspektivisch alters- und geschlechtsspezifisch erfolgen, um u.a. Orientierungen für spezifische Trainingseffekte geben zu können (Borg, 2004; Lamb et al., 2017).

\section{Korrespondenzadresse}

\section{Prof. Dr. Dirk Büsch}

Carl von Ossietzky Universität

Oldenburg, Deutschland

dirk.buesch@uni-oldenburg.de
Danksagung. Wir bedanken uns bei Jelena Braun, Falk Naundorf und Jan Pabst für die Durchführung der Studie 1 und bei Nicolas Bloß für die Realisierung des Online-Fragebogens in Studie 2.

Funding. Open Access funding enabled and organized by Projekt DEAL.

\section{Einhaltung ethischer Richtlinien}

Interessenkonflikt. D. Büsch, T. Utesch und F. Marschall geben an, dass kein Interessenkonflikt besteht.

Studie 1 (Pilotstudie) beruht auf älteren Datensätzen, die unter Berücksichtigung der Deklaration von Helsinki (2013) erhoben wurden. Für Studie 2 (Folgestudie, Online-Umfrage) liegt ein positives Ethikvotum der Universität des Erstautors vor. Von allen beteiligten Patienten liegt eine Einverständniserklärung vor.

Open Access. Dieser Artikel wird unter der Creative Commons Namensnennung 4.0 International Lizenz veröffentlicht, welche die Nutzung, Vervielfältigung, Bearbeitung, Verbreitung und Wiedergabe in jeglichem Medium und Format erlaubt, sofern Sie den/die ursprünglichen Autor(en) und die Quelle ordnungsgemäß nennen, einen Link zur Creative Commons Lizenz beifügen und angeben, ob Änderungen vorgenommen wurden.

Die in diesem Artikel enthaltenen Bilder und sonstiges Drittmaterial unterliegen ebenfalls der genannten Creative Commons Lizenz, sofern sich aus der Abbildungslegende nichts anderes ergibt. Sofern das betreffende Material nicht unter der genannten Creative Commons Lizenz steht und die betreffende Handlung nicht nach gesetzlichen Vorschriften erlaubt ist, ist für die oben aufgeführten Weiterverwendungen des Materials die Einwilligung des jeweiligen Rechteinhabers einzuholen.

Weitere Details zur Lizenz entnehmen Sie bitte der Lizenzinformation auf http://creativecommons.org/ licenses/by/4.0/deed.de.

\section{Literatur}

Abbiss, C., Peiffer, J., Meeusen, R., \& Skorski, S. (2015). Role of ratings of perceived exertion during selfpaced exercise: what are we actually measuring? Sports Medicine, 45(9), 1235-1243. https://doi. org/10.1007/s40279-015-0344-5.

Behr, D., Braun, M., \& Dorer, B. (2015). Messinstrumente in internationalen Studien. GESIS Survey Guidelines. https://doi.org/10.15465/gesis-sg_ 006.

Borg, G. (1982). Psychophysical bases of perceived exertion. Medicine and Science in Sports and Exercise, 14(5), 377-381.

Borg, G. (1998). Borg's perceived exertion and pain scales. Champaign:Human Kinetics.

Borg, G. (2004). Anstrengungsempfinden und körperliche Aktivität. Deutsches Ärzteblatt, 101(15), A106-A1021.

Buckley, J. P., \& Eston, R. G. (2000). Ratings of perceived exertion in braille: validity and reliability in production mode. British Journal of Sports Medicine, 34(4), 297-302.
Carton, R. L., \& Rhodes, E. C. (1985). A critical review of the literature on ratings scales for perceived exertion. Sports Medicine, 2(3), 198-222.

Chen, M. J., Fan, X., \& Moe, S. T. (2002). Criterion-related validity of the Borg ratings of perceived exertion scale in healthy individuals: a meta-analysis. Journal of Sports Sciences, 20, 873-899. https:// doi.org/10.1080/026404102320761787.

Comyns, T., \& Flanagan, E.P. (2013). Applications of the session rating of perceived exertion system in professional rugby union. Strength and Conditioning Journal, 35(6), 70-74.

Egan, A. D., Winchester, J. B., Foster, C., \& McGuigan, M.R. (2006). Using session RPE to monitor different methods of resistance exercise. Journal of Sport Science and Medicine, 5(2), 289-295.

Enoka, R. M., \&Duchateau, J. (2016). Translating fatigue to human performance. Medicine \& Science in Sports \& Exercise, 48(11), 2228-2238. https://doi. org/10.1249/mss.0000000000000929.

Eston, R. G., \& Parfitt, C. G. (2006). Perceived exertion. In N. Armstrong (Hrsg.), Paediatric exercise physiology (S. 275-297).: Elsevier.

Eston, R. G., Lamb, K. L., Bain, A., Williams, M., \&Williams, J. G. (1994). Validity of a perceived exertion scale for children: a pilot study. Perceptual and Motor Skills, 78, 691-697.

Faulkner, J., \& Eston, R. G. (2008). Perceived exertion research in the $21^{\text {st }}$ century: developments, reflections and questions for the future. Journal of Exercise Science \& Fitness, 6(1), 1-14.

Fisher, J., \& Smith, D. (2012). Attempting to better define "intensity" for muscular performance: is it all wasted effort? European Journal of Applied Physiology, 112(12), 4183-4185. https://doi.org/ 10.1007/s00421-012-2463-0.

Fisher, J., Steele, J., Bruce-Low, S., \& Smith, D. (2011). Evidence based resistance training recommendations. Medicina Sportiva, 15(3), 147-162. https://doi.org/10.2478/v10036-0110025-x.

Flick, U. (2002). Qualitative Sozialforschung. Reinbek: Rowohlt.

Foster, C., Boullosa, D., McGuigan, M., Fusco, A., Cortis, C., Arney, B.E., Orton, B., Dodge, C., Jaime, S., Radtke, K., van Erp, T., de Koning, J. J., Bok, D., Rodriguez-Marroyo, J. A., \& Porcari, J.P. (2021). 25 years of session rating of perceived exertion: historical perspective and development. International Journal of Sports Physiology and Performance, 16(5), 612-621. https://doi.org/10.1123/ijspp.2020-0599.

Foster, C., Florhaug, J. A., Franklin, J., Gottschall, L., Hrovatin, L. A., Parker, S., Doleshal, P., \& Dodge, C. (2001). A new approach to monitoring exercise training. Journal of Strength \& Conditioning Research, 15(1), 109-115.

Foster, C., Rodriguez-Marroyo, J. A., \& de Koning, J. J. (2017). Monitoring training loads: the past, the present, and the future. International Journal of Sports Physiology and Performance, 12(Suppl 2), S2-2-S2-8. https://doi.org/10.1123/ijspp.20160388.

Gabbett, T. J. (2016). The training-Injury prevention paradox: should athletes be training smarter and harder? British Journal of Sports Medicine, 50(5), 273-280. https://doi.org/10.1136/bjsports2015-095788.

Gabbett, T.J. (2020). Debunking the myths about training load, injury and performance: empirical evidence, hot topics and recommendations for practitioners. British Journal of Sports Medicine, 54(1), 58-66. https://doi.org/10.1136/bjsports2018-099784. 
Garcin, M., Fleury, A., Mille-Hamard, L., \&Billat, V.(2005). Sex-related differences in ratings of perceived exertion and estimated time limit. International Journal of Sports Medicine, 26, 675-681. https:// doi.org/10.1055/s-2004-830440.

Gaudino, P., laia, F.M., Strudwick, A.l., Hawkins, R.D., Alberti, G., Atkinson, G., \& Gregson, W. (2015). Factors influencing perception of effort (session rating of perceived exertion) during elite soccer training. International Journal of Sports Physiology and Performance, 10,860-864. https://doi.org/10.1123/ijspp.2014-0518.

Gomes, R.L., Lixandrão, M.E., Ugrinowitsch, C., Moreira, A., Tricoli, V., \& Roschel, H. (2020). Session rating of perceived exertion as an efficient tool for individualized resistance training progression. The Journal of Strength \& Conditioning Research. https://doi.org/10.1519/ jsc.0000000000003568.

Haddad, M., Padulo, J., \& Chamari, K. (2014). The usefulness of session rating of perceived exertion for monitoring training load despite several influences on perceived exertion. International Journal of Sports Physiology and Performance, 9, 882-883. https://doi.org/10. 1123/ijspp.2014-0010.

Haddad, M., Stylianides, G., Djaoui, L., Dellal, A., \& Chamari, K. (2017). Session-RPE method for training load monitoring: validity, ecological usefulness, and influencing factors [mini review]. Frontiers in Neuroscience. https://doi.org/10. 3389/fnins.2017.00612.

Halperin, I., \& Emanuel, A. (2020). Rating of perceived effort: methodological concerns and future directions. Sports Medicine, 50(4), 679-687. https://doi.org/10.1007/s40279-019-01229-z.

Hiscock, D. J., Dawson, B., Donnelly, C. J., \& Peeling, P. (2016). Muscle activation, blood lactate, and perceived exertion responses to changing resistance training programming variables. European Journal of Sport Science, 16(5), 536-544. https:// doi.org/10.1080/17461391.2015.1071880.

Hiscock, D. J., Dawson, B., \& Peeling, P. (2015). Perceived exertion responses to changing resistance training programming variables. Journal of Strength \& Conditioning Research, 29(6), 1564-1569. https://doi.org/10.1519/JSC. 0000000000000775.

Hitzschke, B., Holst, T., Ferrauti, A., Meyer, T., Pfeiffer, M., \& Kellmann, M. (2016). Entwicklung des Akutmaßes zur Erfassung von Erholung und Beanspruchung im Sport.Diagnostica, 62(4), 212-226. https://doi.org/10.1026/0012-1924/ a000155.

Hitzschke, B., Kölling, S., Ferrauti, A., Meyer, T., Pfeiffer, M., \& Kellmann, M. (2015). Entwicklung der Kurzskala zur Erfassung von Erholung und Beanspruchung im Sport (KEB). Zeitschrift für Sportpsychologie, 22(4), 146-162. https://doi. org/10.1026/1612-5010/a000150.

Impellizzeri, F. M., Marcora, S. M., \& Coutts, A. J. (2019). Internal and external training load: 15 years on. International Journal of Sports Physiology and Performance, 14(2), 270-273. https://doi.org/10. 1123/ijspp.2018-0935.

Jonkisz, E., Moosbrugger, H., \& Brandt, H. (2012). Planung und Entwicklung von Tests und Fragebogen. In H. Moosbrugger \& A. Kelava (Hrsg.), Testtheorie und Fragebogenkonstruktion (2. Aufl. S. 27-74). Berlin, Heidelberg, New York: Springer. https://doi.org/10.1007/978-3-64220072-4.

Kasai, D., Parfitt, G., Tarca, B., Eston, R., \& Tsiros, M.D. (2021). The use of ratings of perceived exertion in children and adolescents: a scoping review. Sports Medicine, 51(1), 33-50. https://doi.org/ 10.1007/s40279-020-01374-w.

Kellmann, M., Kölling, S., \& Hitzschke, B. (2016). Das Akutmaß und die Kurzskala zur Erfassung von Erholung und Beanspruchung im Sport-Manual. Neuhaus: Sportverlag Strauß.

Kölling, S., \& Kellmann, M. (2020). Current considerations and future directions of psychometric training monitoring of recovery-stress states. Deutsche Zeitschrift für Sportmedizin, 71(2), 29-34.

Kölling, S., Hitzschke, B., Holst, T., Ferrauti, A., Meyer, T., Pfeiffer, M., \& Kellmann, M. (2015). Validity of the acute recovery and stress scale: training monitoring of the German junior national field hockey team. International Journal of Sports Science \& Coaching, 10(2+3), 529-542.

Lamb, K. L., Parfitt, G., \& Eston, R.G. (2013). Effort perception. In N. Armstrong \& W. Van Mechelen (Hrsg.), Paediatric exercise science and medicine (2. Aufl. S. 1-25). Oxford: Oxford University Press. https://doi.org/10.1093/med/9780199232482. 003.0011.2008/2013.

Lamb, K. L., Parfitt, G., \& Eston, R.G. (2017). Effort perception. In N. Armstrong \& W. van Mechelen (Hrsg.), Oxford textbook of children's sport and exercise medicine (3. Aufl. S. 213-222). Oxford: Oxford University Press.

Lienert, G. A., \& Raatz, U. (1994). Testaufbau und Testanalyse. Weinheim: BeltzPVU.

Löllgen, H. (2004). Das Anstrengungsempfinden (RPE, Borg-Skala). Deutsche Zeitschrift für Sportmedizin, 55(11), 299-300.

Mayo, X., Iglesias-Soler, E., \& Fernández-Del-Olmo, M. (2014). Effects of set configuration of resistance exercis on perceived exertion. Perceptual and Motor Skills, 119(3), 825-837. https://doi.org/10. 2466/25.29.PMS.119c30z3.

Micklewright, D., St Clair Gibson, A., Gladwell, V., \& Al Salman, A. (2017). Development and validity of the rating-of-fatigue scale. Sports Medicine, 47(11), 2375-2393. https://doi.org/10.1007/ s40279-017-0711-5.

Morgan, W.P. (1994). Psychological components of effort sense. Medicine and Science in Sports and Exercise, 26(9), 1071-1077.

Noble, B. J., \& Robertson, R. J. (1996). Perceived exertion. Champaign:Human Kinetics.

Pageaux, B. (2016). Perception of effort in exercise science: definition, measurement and perspectives. European Journal of Sport Science, 16(8), 885-894. https://doi.org/10.1080/17461391. 2016.1188992.

Robertson, R. J., Goss, F.L., Rutkowski, J., Lenz, B., Dixon, C., Timmer, J., Frazee, K., Dube, J., \& Andreacci, J. (2003). Concurrent validation of the OMNI perceived exertion scale for resistance exercise. Medicine and Science in Sports and Exercise, 35(2), 333-341. https://doi.org/10 1249/01.MSS.0000048831.15016.2A.

Robertson, R. J., Moyna, N.M., Sward, K. L., Millich, N. B., Goss, F. L., \& Thompson, P. D. (2000). Gender comparison of RPE at absolute and relative physiological criteria. Medicine and Science in Sports and Exercise, 32(12), 2120-2129.

Scott, B. R., Duthie, G. M., Thornton, H. R., \& Dascombe, B. J. (2016). Training monitoring for resistance exercise: theory and applications. Sports Medicine, 46(5), 687-698. https://doi.org/10.1007/ s40279-015-0454-0.

Serafim, T.H.S., Tognato, A.C., Nakamura, P.M., Queiroga, M. R., Nakamura, F.Y., Pereira, G., \& Kokubun, E. (2014). Development of the color scale of perceived exertion: preliminary validation. Perceptual and Motor Skills, 119(3), 884-900. https://doi.org/10.2466/27.06.PMS. $119 \mathrm{c} 28 \mathrm{z} 5$.

Williams, J.G., Eston, R., \& Furlong, B. (1994). CERT: a perceived exertion scale for young children Perceptual and Motor Skills, 79, 1451-1458. 